\title{
QSTR study of four synthesized nitrobenzene derivatives
}

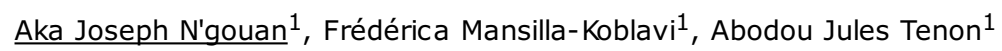 \\ ${ }^{1}$ UFR SSMT Université Félix Houphouët Boigny Abidjan Côte D'Ivoire, Abidjan, Ivory Coast \\ E-mail: josephngouan@yahoo.fr
}

Nitrobenzene derivatives are widely used as reactional intermediates in the synthesis of many chemicals in medicine, industry, and agriculture. This study is based on four synthesized nitrobenzene derivatives which crystal structures have been determined. As it is known, nitro compounds as well as their metabolites are hazardous chemicals, harmful for human and environment. Some of them have mutagenic or carcinogenic activity and others can cause aquatic species growth inhibition. So, for a best use of these new compounds, we have decided to have a best insight of their possible toxicity. An important field of predictive toxicity is the development of quantitative structure toxicity relationship (QSTR) for modeling chimicals toxicity. Applications of such mathematical models are numerous and increasing because results of QSTR are closed to those obtained experimentally. Two series of nitro compounds which exhibit toxicity, particularly carcinogenic activity (TD50) and growth inhibitory activity (IG50) were used as targets for a mathematical model building. calculations of descriptors were then performed over all the molecules, both the synthesized ones and those used for the model building, after minimizing their structure with CS MOPAC Pro software using semi-empirical Austin Model 1 (AM1) algorithm. Quantitative structuretoxicity relationship (QSTR) was established by correlating the toxicity and the calculated descriptors. The best models were retained according to valid statistical parameters.Both equations below, deriving from PLS regression model, express the toxicity via the descriptors included, octanol/water partition $(\log \mathrm{P}(\mathrm{o} / \mathrm{w})$, SlogP), number of oxygen atom (a_no), weight, dipole moment (MNDO_dipol) and the Homo energy (MNDO_HOMO): Carcinogenic activity = 0,631 + 0,533.a_nO + 0,175. $\log \mathrm{P}(\mathrm{o} / \mathrm{w})+9,889 . \mathrm{MNDO}$ _dipol Growth inhibitory activity $=0,717$. SlogP $+0,125 . \mathrm{MNDO}$ HOMO $+0,536$. Weight The statistical parameters, respectively $\left(\mathrm{MCE}=0,012, \mathrm{R}^{2}=0,954, \mathrm{Q}^{2}=0,942\right)$ and $\left(\mathrm{MCE}=0,011, \mathrm{R}^{2}=0,958, \mathrm{Q}^{2}=0,946\right)$ are satisfactory and the toxicity measured are well predicted by the two models. The calculations performed allowed us to conclude that the compounds understudied are all potentially carcinogenic and two of these new compounds are more likely inhibitors of aquatic organisms growth.

[1] Patai, S.(1982). The Chemistry of Amino, Nitroso, and Nitro Compounds and Their Derivatives, edited by John Wiley \& Sons Inc: New York.

[2] Neilson A.H. and Allard A.S. (2008). Environmental Degradation and Transformation of Organic Chemicals, edited by Boca Raton, Florida: CRC Press.

[3] N'gouan, A. J. (2014).Contribution à l'étude de l'activité biologique de composés dérivés du nitrobenzène. Doctoral thesis. Université Félix Houphouët Boigny, Abidjan, Côte d'ivoire.

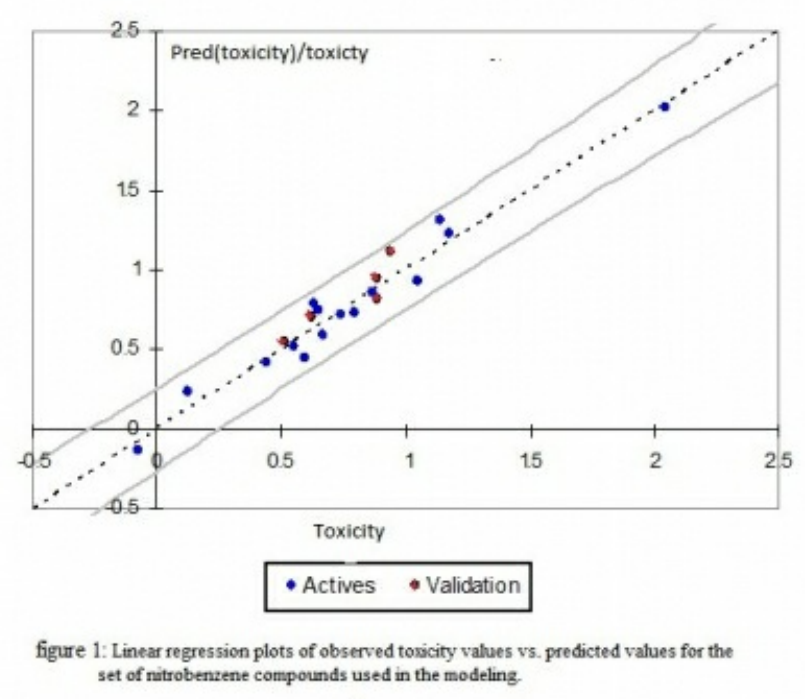

Keywords: Nitrobenzene derivatives, structure-toxicity relationship, carcinogenic. 